\title{
Dynamic RWA in WDM optical network
}

\author{
Harpreet Kaur $^{1}$ and Munish Rattan ${ }^{2}$ \\ ${ }^{1} A P, B B S B E C$, Fatehgarh Sahib,Punjab,India. \\ ${ }^{2} A P$, GNDEC,Ludhiana,Punjab,India.
}

\begin{abstract}
As optical wdm networks are becoming the backbone building blocks of twenty first century's networks due to their capacity of carrying huge bandwidth. So evaluating the performance of optical wdm networks and optimizing it, is need of the day. For this the problem of routing and wavelength assignment is of significance importance. Researchers have done extensive simulations for both static and dynamic routing and wavelength problem. In this paper we have presented various algorithms for solving the dynamic RWA problem in literature.Also we have prepared a optical wdm network using which RWA problem is analysed.
\end{abstract}

\section{Introduction}

Nowadays optical wdm networks are emerging technologies networks that can provide routing, grooming, and restoration at wavelength level [2]. WDM technology of crucial importance for satisfying the ever increasing capacity requirements in telecommunication networks. Wavelength routed WDM networks can exploit the large bandwidth of optical fibers by dividing it among different wavelengths. These networks are equipped with configurable WDM nodes which enable us to set up and tear down all-optical channels, called light paths, between pairs of nodes. Given a network and a set of light path requests, the Routing and Wavelength Assignment (RWA) problem attempts to route each light path request, and to assign wavelengths to these routes subject to the following constraints $[1,13]$.

The RWA problem can be categorized into two types, static RWA (SRWA) and dynamic RWA (DRWA). In a wavelength-routed optical network (WRON), the traffic demands can be either static or dynamic In static RWA, all the connection requests are known in advance and the objective of RWA is to assign routes and wavelengths to all the light paths globally while keeping the amount of network resource required minimal [5].In a dynamic traffic pattern, a light path is set up for each connection request as it arrives and it is released after some finite amount of time. One of the challenges involved in designing wavelength routed networks with dynamic traffic demands is to develop efficient algorithms and protocols for light paths establishing [7, 8, 10 and 11].

In all-optical WDM networks, each of the connections going through a link is assigned a wavelength. If a connection is assigned the same wavelength on all links along its routing path, the signal is able to travel from the source node to the destination node using the same wavelength. In case that the connection has to be

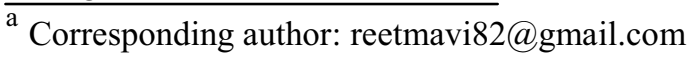

assigned two or more wavelengths on different links, one or more nodes along its routing path must have the capability of converting the signal from one wavelength to another wavelength. If all nodes along the routing path of a connection are incapable of converting a wavelength to another wavelength, the connection must be assigned the same wavelength on all links along its path. Otherwise, the connection is blocked. This is known as the wavelength continuity constraint. A wavelength which is available on all links along a routing path is referred to as a common available wavelength along the routing path[6]. In addition, light paths that share a common physical link cannot be assigned the same wavelength. This is called the wavelength clash constraint $[13,1]$.

There are number of algorithms available in literature for dynamic routing and wavelength assignment problem and rsearchers too have used optimization techniques and have formulated various new algorithms to optimize the performance of optical wdm network in terms of dynamic wavelength and assignment problem. In this paper we have presented the review of various techniques available for solving dynamic routing and wavelength problem.

\section{ALGORITHM USED FOR DRWA}

\subsection{Fixed Path Routing}

It uses only one route from source end to destination .In this approach the network resources are not properly utilized.[1]

\subsection{Alternate Path Routing}


It uses alternate paths from source to a destination .If the first is unavailable then second path is used.[15]

In dynamic routing in DRW1 algorithm no weight assignment is done all links have same weight. First all possible paths between the source and destination is calculated then request is routed over the shortest path if wavelength is available else alternate shortest path is used. In DRW2 less weight is assigned to nodes which are used less. As the request arrived it is routed to the nodes having less weight if wavelength is available if not then request is routed through next higher weight nodes and the process is repeated. In DRW3 algorithm less weight is assigned to the nodes which are less used and are nearest and then routing of request is done through routes having minimum weight if wavelength is available else next higher weight is tried and the process is repeated [9] Another algorithm is proposed in literature which first selects $\mathrm{K}$ edge disjoint least weighted paths according to the current network state and then one such path is considered to route the request taking into account not only the load, length of the path but also number of transceivers in the nodes along the path.after this an auxiliary graph is generated and request is routed on least weight route on the graph and wavelength is assigned for the same.[14]

\subsection{Fixed alternate shortest Path routing with least Priority wavelength assignment Strategies}

In this algorithm the wavelength information table is made according to the use of the each wavelength in the network then the lightpath is set on shortest or alternate shortest path with the least used wavelength if available else the route call is blocked.[16]

\subsection{Exhaust Algorithm}

It searches all the possible routes between source and destination and then finally selects the best route. So utilizing this route provides the flexibility of selecting any route according to the wavelength available.Source Initiated Reservation Algorithm In this algorithm the network resources are utilized by reducing control overhead .In this the route table is generated first and then resource and wavelength availability is updated .Next the route table is arranged according to minimum hops and maximum wavelengths available and route is searched according to that. If wavelength allotted the request is processed else the request is blocked and then whole process is repeated.[15]

\subsection{Traffic Intensity Based Fixed -Alternate Routing}

In this algorithm the traffic is splitted for each source destination pair onto the disjoint routing paths by solving the nonlinear multicommodity flow optimization problem. Further the routing path between end users is sorted in decending order according to the amount of traffics of source destination pair assigned to the routing paths. For the request arrived this algorithm tries the multiple routing paths between the particular source destination pair one by one in the sorted order to establish a connection until the request is processed successfully otherwise the request is blocked .Results proved that this algorithm have lower blocking probability as compared if sorting is done according to the hop count instead of traffic intensities.[3]

\subsection{Priority and Maximum Revenue based Routing}

In this algorithm two types of requests are kept in two light path request matrixes namely high priority matrix (HPM) and low priority matrix (LPM).High priority matix requests are entertained first before LPM requests. According to this algorithm the light paths in HPM must be fulfilled and revenue for the same is fixed while the light paths in the LPM can be set up selectively to optimize revenue when there is not enough uresource to accommodate all.[12]

Multiobjective integer linear program is proposed in literature for maximizing throughput and solving traffic engineering in wdm optical networks.This program collectively maximizes the network output,minimizes the resource consumption and achieves load balancing by minimizing the maximum link utilization. This algorithm solves the multiobjective problem as an e-constraint problem upper bounding the per route resource consumption and maximizing the throughput by routing through multiple widest paths.Simulations results obtained reveals that solving multiobjective problem by ILP (Integer linear Programming) yield promising results.[4]

\section{Simulation Results}

For analyzing the problem of routing and wavelength assignment we have prepared a wdm network in which each link is bidirectional and taken eight wavelengths per link. For routing shortest path algorithm is used and first fit for wavelength assignment. The traffic considered is dynamic in nature which follows the poisson process. We have analysed and calculated the thoughput for two types of applications i.e FTP and email .The simulation is carried on opnet modeler.

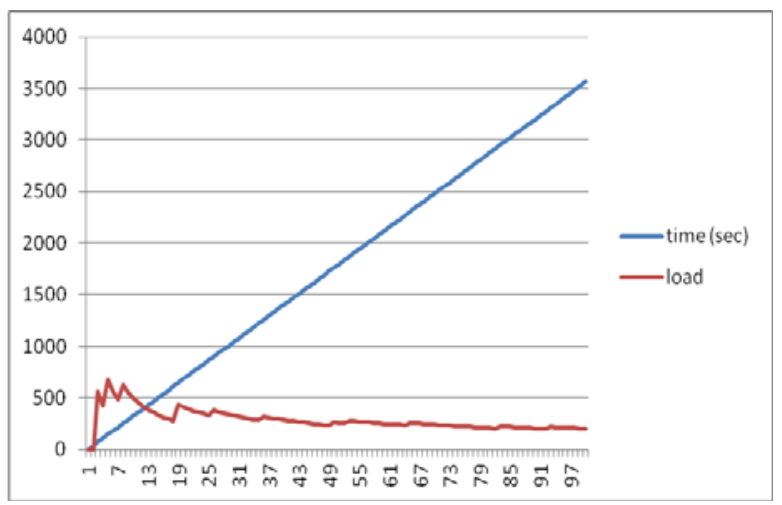

Figure 1. Load versus time for email application. 


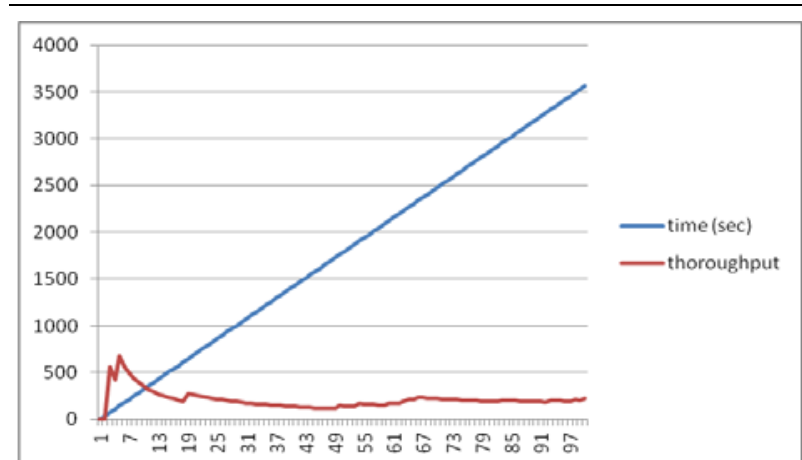

Figure 2. Throughput versus time..

Table 1. Data sheet for load and throughput.

\begin{tabular}{|c|c|c|}
\hline & Data Collected & \\
\hline time (sec) & load & throughput \\
\hline 0 & 0 & 0 \\
\hline 36 & 0 & 0 \\
\hline 72 & 565.33333 & 565.3333333 \\
\hline 108 & 424 & 424 \\
\hline 144 & 678.4 & 678.4 \\
\hline 180 & 565.33333 & 565.3333333 \\
\hline 216 & 484.57143 & 484.5714286 \\
\hline 252 & 622.75 & 437.25 \\
\hline 288 & 553.55556 & 388.6666667 \\
\hline 324 & 498.2 & 349.8 \\
\hline 360 & 452.90909 & 318 \\
\hline 396 & 415.16667 & 291.5 \\
\hline 432 & 383.23077 & 269.0769231 \\
\hline 468 & 355.85714 & 249.8571429 \\
\hline 504 & 332.13333 & 233.2 \\
\hline 540 & 311.375 & 218.625 \\
\hline 576 & 293.05882 & 205.7647059 \\
\hline 612 & 276.77778 & 194.3333333 \\
\hline 648 & 435.15789 & 278.9473684 \\
\hline 684 & 413.4 & 265 \\
\hline 720 & 393.71429 & 252.3809524 \\
\hline 756 & 375.81818 & 240.9090909 \\
\hline 792 & 359.47826 & 230.4347826 \\
\hline 828 & 344.5 & 220.8333333 \\
\hline 864 & 330.72 & 212 \\
\hline 900 & 379.15385 & 207.9230769 \\
\hline 936 & 365.11111 & 200.2222222 \\
\hline 972 & 352.07143 & 193.0714286 \\
\hline 1008 & 339.93103 & 186.4137931 \\
\hline 1044 & 328.6 & 180.2 \\
\hline 1080 & 318 & 174.3870968 \\
\hline 1116 & 308.0625 & 168.9375 \\
\hline 1152 & 298.72727 & 163.8181818 \\
\hline
\end{tabular}

\begin{tabular}{|c|c|c|}
\hline 1188 & 289.94118 & 159 \\
\hline 1224 & 281.65714 & 154.4571429 \\
\hline 1260 & 318 & 153.1111111 \\
\hline 1296 & 309.40541 & 148.972973 \\
\hline 1332 & 301.26316 & 145.0526316 \\
\hline \multirow[t]{2}{*}{1368} & 293.53846 & 141.3333333 \\
\hline & Data Collected & \\
\hline time $(\mathrm{sec})$ & load & throughput \\
\hline 1404 & 286.2 & 137.8 \\
\hline 1440 & 279.21951 & 134.4390244 \\
\hline 1476 & 272.57143 & 131.2380952 \\
\hline 1512 & 266.23256 & 128.1860465 \\
\hline 1548 & 260.18182 & 125.2727273 \\
\hline 1584 & 254.4 & 122.4888889 \\
\hline 1620 & 248.86957 & 119.826087 \\
\hline 1656 & 243.57447 & 117.2765957 \\
\hline 1692 & 238.5 & 114.8333333 \\
\hline 1728 & 233.63265 & 112.4897959 \\
\hline 1764 & 262.88 & 144.16 \\
\hline 1800 & 257.72549 & 141.3333333 \\
\hline 1836 & 252.76923 & 138.6153846 \\
\hline 1872 & 278 & 138 \\
\hline 1908 & 274.81481 & 164.8888889 \\
\hline 1944 & 269.81818 & 161.8909091 \\
\hline 1980 & 265 & 159 \\
\hline 2016 & 260.35088 & 156.2105263 \\
\hline 2052 & 255.86207 & 153.5172414 \\
\hline 2088 & 251.52542 & 150.9152542 \\
\hline 2124 & 249.1 & 174.9 \\
\hline 2160 & 245.01639 & 172.0327869 \\
\hline 2196 & 241.06452 & 169.2580645 \\
\hline 2232 & 238.92063 & 191.8095238 \\
\hline 2268 & 236.84375 & 213.65625 \\
\hline 2304 & 257.66154 & 212 \\
\hline 2340 & 255.36364 & 232.8787879 \\
\hline 2376 & 251.55224 & 229.4029851 \\
\hline 2412 & 247.85294 & 226.0294118 \\
\hline 2448 & 244.26087 & 222.7536232 \\
\hline 2484 & 240.77143 & 219.5714286 \\
\hline 2520 & 237.38028 & 216.4788732 \\
\hline 2556 & 234.08333 & 213.4722222 \\
\hline 2592 & 230.87671 & 210.5479452 \\
\hline 2628 & 227.75676 & 207.7027027 \\
\hline 2664 & 224.72 & 204.9333333 \\
\hline 2700 & 221.76316 & 202.2368421 \\
\hline
\end{tabular}




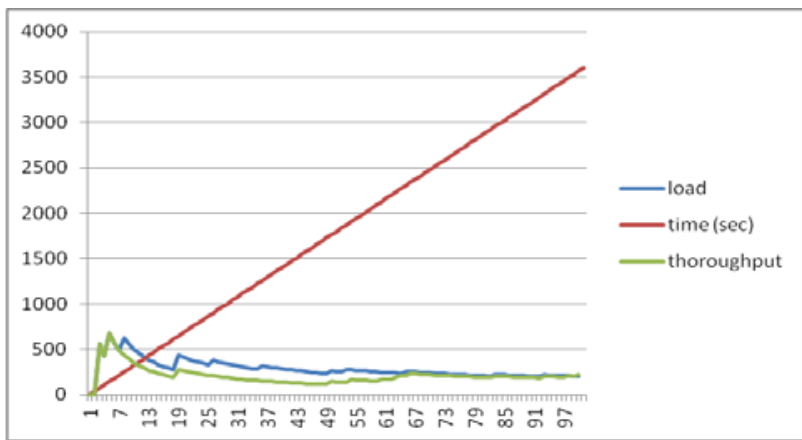

Figure 3. Throghput versus time and load for email.

\section{Conclusion}

The traffic is dynamic in nature so the load is varying w.r.t time. Throughput is the performance metric taken in this paper which is observed lesser compared to the load offered to the network which indicates the blockage of requests offered to the network. The throughput and blocking of the network can be optimized with some nature inspired technique in future.

\section{References}

1. Mukherjee B., Optical Communication Networks (McGraw-Hill, New York, 1997)

2. Chen, M. T., Lin, B. M. T. and Tseng, S. S. J. Engineering applications Of Artificial Intelligence, 24,2(2011)

3. Chun, L. H., Wei, W. S., and Tsai, C. P. , Journal of Light wave technology, 26,2(2006)

4. Crichigno, J., Xie, C., Shu, W., Wu, M. Y. and Ghani, N., Proceedings of International Conference Asilomar, Pacific Grove, CA (2009)

5. Zang, H., .Jue, J.P.., and Mukherjee, B. Optical Networks Magazine, 1, 1(2000).

6. Chlamtac I., Ganz A., and Karmi G. , IEEE Transactions on Communications, $40,7(1992$

7. Kaminow I.P. et al. ,J. Select. Areas Commun. 14, 6, (1996)

8. Ramaswami R., Sivarajan K. N., J. Select. Areas Commun. 14, 6(1996)

9. Randhawa, R. and Sohal, J.S. J. Optik, 121, 8(2010)

10. Ramaswami R., Sivarajan K. N., IEEE/ACM Transactions on Networking,3,5(1995)

11. V.Acimovic-Raspopovi, G. Markovic, Invited paper, Proc.of TELFOR Conference, Belgrade (2004)

12. Wang, Y., Cheng, T. H., and Ma, M., (2007) Proceedings of International Conference on Research Innovation Vision of the Future, Hanoi (2007)

13. X. Jia, X.-D.Hu, D.-Z. Du, Multiwavelength Optical Networks, Kluwer Academic Publishers, Norwell, MA (2002)

14. Ye, Y., Chai, T. Y., Chen, T. H. and Lu, C. J. Computer Communications 29, 15(2006)

15. Zaidi, M. H., Mahmood, A., Proceedings of International Conference INMIC, Islamabad (2003)

16. Bhatt U. R. and Tokekar S., J. optik ,122(2010) 